\title{
Signal de-noising in gear pitting fault identification by an improved singular value decomposition method
}

\author{
Xintao Zhou' ${ }^{1}$ Yahui Cui ${ }^{1}$ Longlong Li · Lihua Wang · Xiayi Liu • Baofeng Zhang \\ Received: 9 October 2019 / Accepted: 20 February 2020 / Published online: 21 March 2020 \\ (c) The Author(s) 2020
}

\begin{abstract}
In this research a new method of improved singular value decomposition (ISVD) is proposed for the vibration signal de-noising of gear pitting fault identification. In this method, the delay time $\tau$ and embedding dimension $m$ of the Hankel matrix for SVD are optimized by autocorrelation function and Cao's algorithm respectively. Simulation and experiments are conducted to demonstrate the method. In the simulation, the ISVD method is employed to de-noise the artificial vibration signal in a mathematical model of gear pitting fault, the result demonstrates the signal-noise ratio (SNR) value is $\mathrm{SNR}=31.3 \mathrm{~dB}$, and the root-mean-square error (RMSE) value is RMSE $=0.34$. In the experiment, the ISVD method is adopted to de-noising the vibration signal of gear pitting fault identification, the results demonstrate SNR is SNR $>45 \mathrm{~dB}$, and the RMSE value is RMSE $<0.4$ of the fault characteristic signals at each measuring point position. The results of simulation and experiment show, the ISVD method is efficient to de-noise the vibration signal of gear pitting fault.
\end{abstract}

\section{Signalentrauschen in Zahnrad-Lochfehler-Identifikation durch eine verbesserte Singular-Value-Zersetzungsmethode}

\section{Zusammenfassung}

In dieser Forschung wird eine neue Methode zur verbesserten Singularwertzersetzung (ISVD) für die Schwingungssignalentnosierung von Zahnrad-Lochfehler-Identifikation vorgeschlagen. Bei dieser Methode werden die Verzögerungszeit und die Einbettungsdimension $m$ der Hankel-Matrix für SVD durch die Autokorrelationsfunktion bzw. den Cao-Algorithmus optimiert. Simulationen und Experimente werden durchgeführt, um die Methode zu demonstrieren. In der Simulation wird die ISVD-Methode verwendet, um das künstliche Schwingungssignal in einem mathematischen Modell von Zahnrad-PittingFehlern zu entrauschen; das Ergebnis zeigt den Signal-Rausch-Verhältnis-Wert (SNR) sNR = 31,3 dB und der RMSE-Wert (Root-Mean-Square Error) ist RMSE = 0,34. Im Experiment wird die ISVD-Methode zum Entrauschen des Schwingungssignals der Schaltfehleridentifikation von Zahnrädern angewandt, die Ergebnisse zeigen, dass SNR SNR > $45 \mathrm{~dB}$ ist, und der RMSE-Wert ist RMSE -0,4 der Fehlerkennzeichensignale an jeder Messpunktposition. Die Ergebnisse der Simulation und des Experiments zeigen, dass die ISVD-Methode effizient ist, um das Schwingungssignal von Zahnrad-Pitting-Fehlern zu entlärmen.

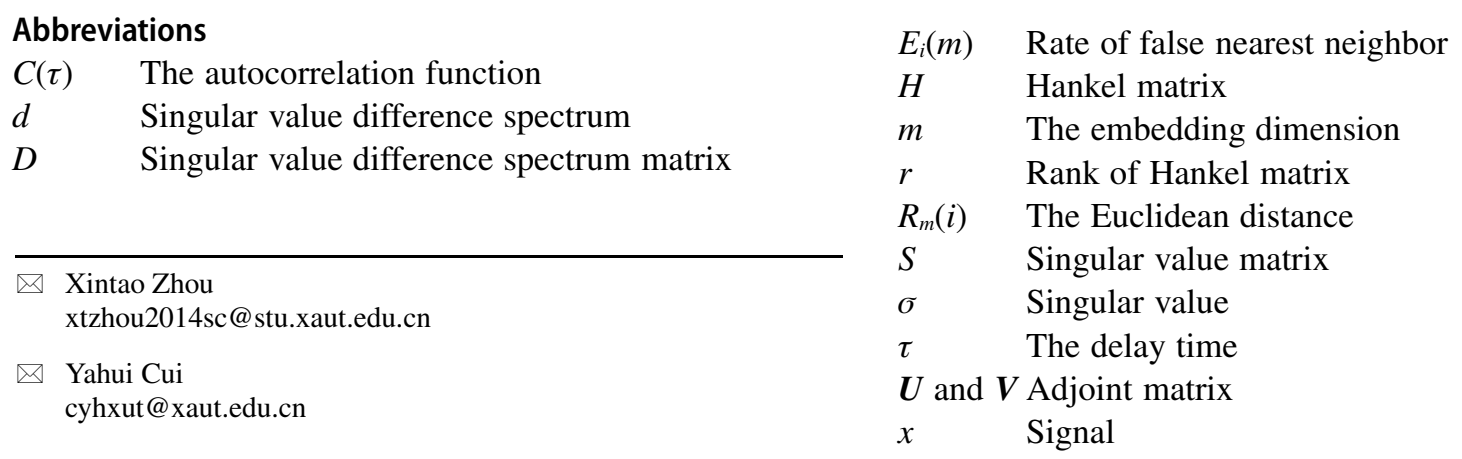

1 School of Machinery and Precision Instrument Engineering, Xi' an University of Technology, 710048 Xi'an, China 


\section{Introduction}

Gears are critical transmission components in rotating machinery with extensive application field especially in modern industrial areas. The whole machinery's performance and production efficiency are directly affected by the working state of gears [1]. The stable operation is directly affected by the state of gear motion whether the gear run normally or not $[2,3]$. Therefore, it is very crucial to identify the early faults before the gears develop into serious state. However, in practical applications, it is difficult to predict the formation and development of gear fault in advance because while the vibration around the gearbox is perceived, the gear fault has been a severe condition. Up to now, the research of the gear failure mechanism in early and middle condition is not mature. For the practical applications, it is important to develop the research of gear failure on vibration characteristics of gear box.

Recently, many theoretical types of research are developed on the vibration of gearbox by gear failure, few type are based on practical application. And there is a certain gap between theory and practical application. Generally, fault detection of gear teeth is the collection of fault signal samples site, and the identification of the gear fault type according to the information by the signal collection [4]. Since the measured signal is interfered with strong noise background, de-noising for the extraction of useful characteristics from the original signal is the first important stage. At present, the common methods of signal de-noising are various, such as Empirical mode decomposition method (EMD) [5, 6], Local mean decomposition method (LMD) [7, 8], Singular value decomposition method (SVD) [9-11] wavelet analysis method [12-14]. In practical, the methods are limited by some problems. EMD is affected by mode aliasing which is occurred frequently in the decomposition process, and the quality of signal decomposition is reduced in the condition $[15,16]$. LMD also generates mode aliasing which makes the decomposition and extraction of fault features incomplete, and lead to decomposition failure on the whole time scale [17-19]. The effect of Wavelet de-noising method is depended on the calculation and selection of reasonable parameters $[20,21]$.

Beside the de-noising methods above, the singular value decomposition (SVD) is a better method to deal with problems of mode aliasing and endpoint effect. The singular value of effective feature is distinguished from original signal by SVD which is based on the singular value matrix of the characteristic signal. In the method, characteristic information of the noise signal is eliminated, the useful characteristic signal is reconstructed. As it is invariant, stable and effective for the de-noising, SVD is applied to the filtering of nonlinear signals, such as practical application of de-noising in fault identification of gear fault [22].
However, the accuracy of de-noising in fault identification by SVD is depended on the calculations of $\tau$ and $m$, which is highly subjective and unreliable in theory at present. Although suitable parameters of $\tau$ and $m$ are calculated by a new method which is called $k$-SVD, the determination of $k$ is restricted by other conditions, and affects the de-noising effect of SVD [23, 24].

It is urgency to reasonably determinate the values of $\tau$ and $m$ in SVD respectively. For the value of $\tau$, although is appropriate to any value in theory, the determination in practical applications is directly affected by the vibration signal. In the case that $\tau$ is too small, the correlation is concentrated to between different elements of the delay vector, and the reconstructed phase space is meaningless. Contrary, while $\tau$ is too large, it leads to loss of noise delay coordinates of mutual information elements, and the reconstructed phase space is unable to reflect the initial dynamic behavior. The parameter $m$ controls the phase space, determines the accuracy of de-noising and identification. While $m$ is large, the phase space is too large, and makes the calculation tedious and complex. Contrary, while $m$ is small, the reconstructed information is unrealizable for the extraction from mutation signals [25, 26].

In order to improve the SVD for de-noising, an improved singular value decomposition (ISVD) is developed based on autocorrelation function with Cao's algorithm for the parameter of $\tau$ and $m$. For the determination of $\tau$, the autocorrelation function is closely related to the shape of the attractor in the reconstructed phase space and approximated the principal element direction of the signal system. Empirically, while the decay of autocorrelation function is approximated to $1 / e$, the optimal delay time is obtained [35]. For the determination of $m$, Cao's algorithm which is based on the False Nearest Neighbor (FNN) method, makes the calculation of $m$ only related to $\tau$, and overcomes the influence of various thresholds on $m$ [27, 28].

In this paper, ISVD is proposed for the vibration signal de-noising of gear pitting fault identification. Firstly, the parameter of $\tau$ and $m$ of the Hankel matrix for SVD are optimized by autocorrelation function and Cao's algorithm respectively. Secondly, simulation of ISVD by autocorrelation function and Cao's algorithm is conducted to verify the proposed method. Thirdly, the experiment of tooth surface pitting failure is conducted by ISVD method to verify the effectiveness of the proposed method.

\section{Theory of the SVD method}

Fig. 1 shows the flowchart of ISVD for de-noising. In the flow, the optimal $\tau$ is calculated by the autocorrelation function method of fault signal sequence; $m$ is calculated by the Cao's algorithm of fault signal phase space, based on them, 
Fig. 1 Flowchart of the ISVD method for de-noising

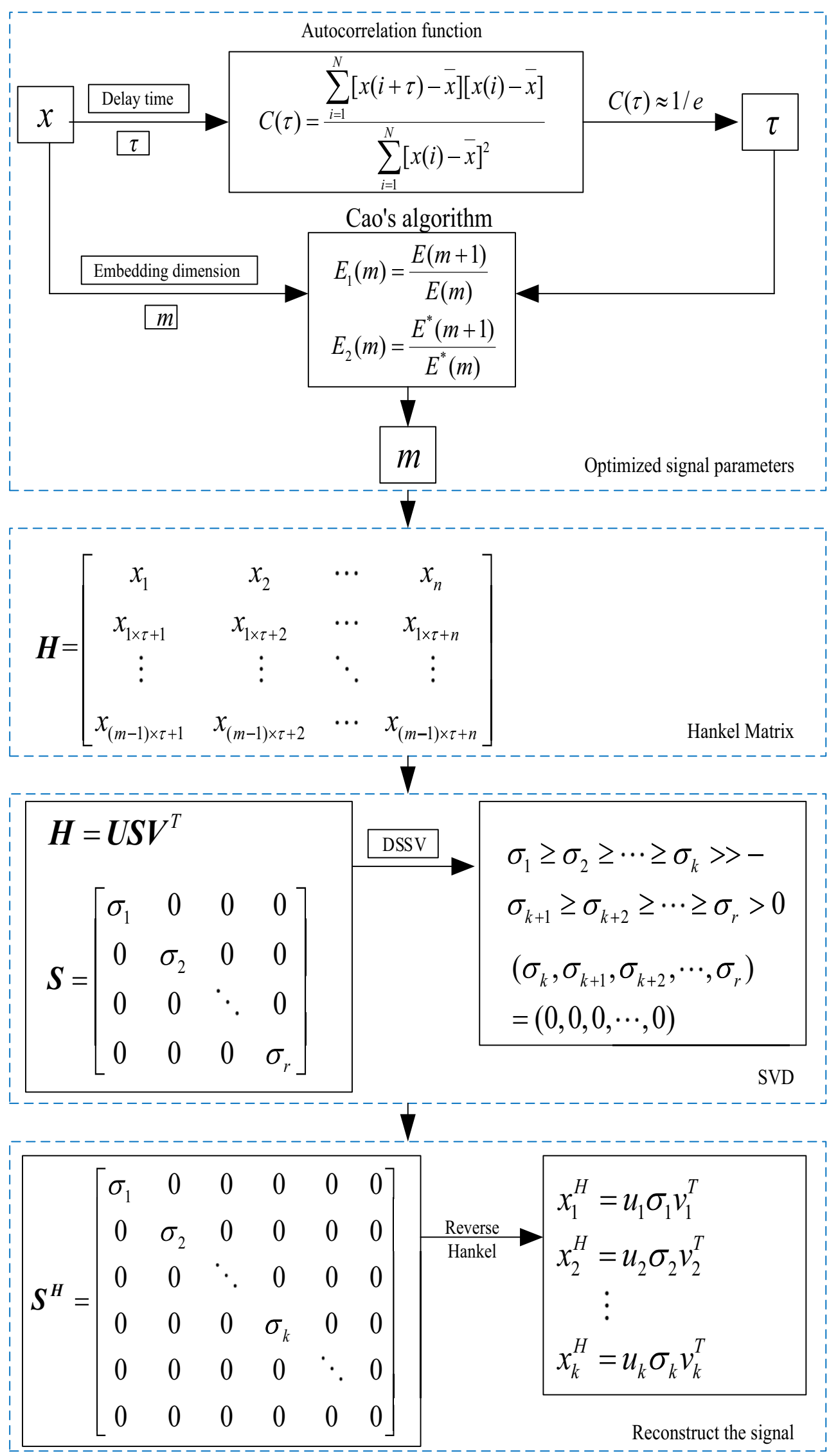


the phase space of the effective characteristic signals and noise signals are obtained respectively; then the optimal de-nosing order is calculated by the singular value difference spectrum (DSSV) method of the characteristic signal; finally, the inverse operation of Hankel matrix is used to reconstruct the characteristic signal after de-noising. It retains the effective signal value and rid the noise signal value, then reconstructs the effective signal in a suitable order. Essentially, SVD is a method of matrix orthogonalization in the mathematical view, which decomposes the given matrix into two matrixes $\boldsymbol{U}^{m \times m}$ and $\boldsymbol{V}^{n \times n}$, as shown: [29-32].

$\boldsymbol{H}=\boldsymbol{U} \boldsymbol{S} V^{T}$

where $\boldsymbol{S}$ is the singular value matrix of the real matrix $\boldsymbol{H}$, in $\boldsymbol{S}, \sigma_{i}$ is the singular value of the real matrix $\boldsymbol{H}$, and $\sigma_{1} \geq \sigma_{2} \geq \cdots \geq \sigma_{i} \geq \sigma_{r} \geq 0$, where $r$ is the rank of the matrix $\boldsymbol{H}$.

For the signals $x=\left(x_{1}, x_{2}, \cdots, x_{m}\right)$, the de-noising is described as follows:

(i) Establishment of a Hankel matrix $\boldsymbol{H}$ based on $\boldsymbol{X}, \boldsymbol{H}$ is expressed by Eq. 2 .

$\boldsymbol{H}=\left[\begin{array}{cccc}x_{1} & x_{2} & \cdots & x_{n} \\ x_{1 \times \tau+1} & x_{1 \times \tau+2} & \cdots & x_{1 \times \tau+n} \\ \vdots & \vdots & \ddots & \vdots \\ x_{(m-1) \times \tau+1} & x_{(m-1) \times \tau+2} & \cdots & x_{(m-1) \times \tau+n}\end{array}\right]$

where $m$ is the embedded dimension, $\tau$ is delay time, $N$ is the length of the signal, and $n=N-(m-1) \times \tau$.

(ii) Calculation of the orthogonal matrix $S=\operatorname{diag}\left(\sigma_{1}, \sigma_{2}, \ldots\right.$, $\left.\sigma_{r}\right)$ on $\boldsymbol{H}$ by SVD.

(iii) Ordination of the elements such as $\sigma_{1}, \sigma_{2}, \ldots, \sigma_{r}$, in $S$, to satisfy the condition of $\sigma_{1} \geq \sigma_{2} \geq \ldots \sigma_{k} \gg$ $\sigma_{k+1} \geq \sigma_{k+2} \geq \ldots \geq \sigma_{r}>0$.

(iv) Finding out the mutation point $k$ in Step. (iii), retaining the elements from $\sigma_{1}$ to $\sigma_{k}$, and defining the other elements equal to 0 as $\left(\sigma_{k+1}, \sigma_{k+2}, \ldots, \sigma_{r}\right)=(0,0, \ldots, 0)$.

(v) Reconstruction of the singular value matrix according to the order $k$ in Step.(iv), to achieve the characteristic signal of de-noising.

$k$ is investigated by Singular values difference spectrum (DSSV) $\boldsymbol{D}$ and the elements in $\boldsymbol{D}$ is $d=\sigma_{i}-\sigma_{i+1}$, where $i=r-1$. The DSSV sequence $\boldsymbol{D}_{i}$ is constructed shown in Eq. 3.

$D_{i}=\left\{d_{i} \mid i=1,2, \cdots, r-1\right\}$
While the difference spectrum of singular value is positioned on the mutation point, the spectrum value $d_{i}$ is the maximum in the whole sequence $\boldsymbol{D}_{i}$. It is expressed by:

$d_{\max }=\max D_{i}=\max \left\{d_{i} \mid i=1,2, \cdots, r-1\right\}$

Based on of Eq. 4, the sequence number corresponding to the maximum SVDS $d_{\max }=\operatorname{Max}\left(\boldsymbol{D}_{i}\right)$ is calculated of the signal. According to the above principle, the new singular value matrix $\boldsymbol{S}_{\mathrm{New}}$ is obtained from the effective characteristic signal, as shown in Eq. 5:

$S_{\mathrm{New}}=\operatorname{diag}\left(\sigma_{1}^{H}, \sigma_{2}^{H}, \sigma_{3}^{H}, \cdots, \sigma_{i}^{H}, 0, \cdots, 0\right)$

where, $\sigma^{H}$ is the singular value left over.

(vi) Reconstruction of characteristic signals $X_{i}^{H}$, show as follow:

$X_{i}^{H}=u_{1}^{H} \sigma_{1}^{H} v_{1}^{H}+u_{2}^{H} \sigma_{2}^{H} v_{2}^{H}+\cdots+u_{h}^{H} \sigma_{i}^{H} v_{h}^{H}$

where, $u_{i}^{H}$ is element of the matrix $\boldsymbol{U}, v_{i}^{H}$ is element of the matrix $\boldsymbol{V}$.

\section{Characteristic parameters of ISVD method}

In this section, the two parameters of $\tau$ and $m$ are optimized by the combination of autocorrelation and the advantages of Cao's algorithm.

\subsection{The parameter of delay time $\tau$}

A measured time series is $x(t)$, and the autocorrelation function of the sequence after normalization processing is shown in Eq. 7 [33, 34]:

$C(\tau)=\frac{\sum_{i=1}^{N}[x(i+\tau)-\bar{x}][x(i)-\bar{x}]}{\sum_{i=1}^{N}[x(i)-\bar{x}]^{2}}$

where, $N$ is sample size, $\tau$ is delay time, $\bar{x}$ is average value of the samples,

$\bar{x}=\frac{1}{N} \sum_{i=1}^{N} x(i)$.

The research shows, the corresponding time is selected while the value of the autocorrelation function exactly decayed to $1 / e$ by the autocorrelation function method, as the optimal value of the $\tau$ of the effective phase space feature vector [35]. 


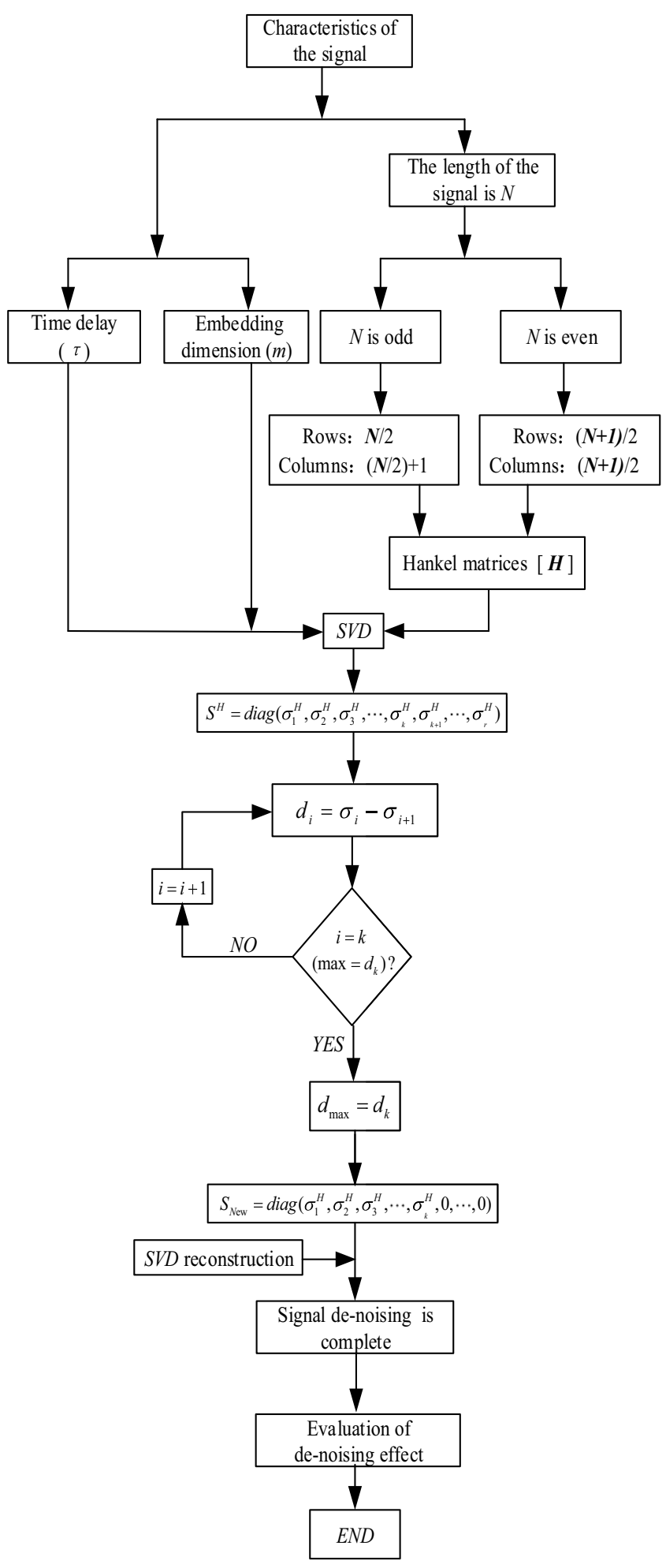

Fig. 2 Flowchart of the signal de-noising

\subsection{The parameter of embedded dimension $m$}

The principle of Cao's algorithm is expressed as the list [36]:

(i) On the definition, an $m$-dimension "embedding space", the time series vector $\vec{X}_{m}(i)$ at the $i$ th phase point is defined as

$\vec{X}_{m}(i)=$

$[x(i), x(i+\tau), x(i+2 \tau), \cdots, x(i+(m-1) \tau)]^{T}$

where, $x(i)$ is the signal corresponding to the time series; $i$ is the time-series sequence number, $i=1,2, \ldots$, $N-(m-1) \tau$.

Then the Euclidean distance $R_{m}(i)$ from $\vec{X}_{m}(i)$ to the nearest neighbor $\vec{X}_{m}^{N N}(i)$ is calculated as:

$R_{m}(i)=\left\|\vec{X}_{m}(i)-\vec{X}_{m}^{N N}(i)\right\|$

where, $\|\bullet\|$ is the $\infty$-norm, and all the normalizations followed are $\infty$-norm.

(ii) While the $m$-dimensional phase space is extended to $m+1$, similarly, the $i$ th phase point is $\vec{X}_{m+1}(i)$, and the Euclidean distance $R_{m+1}(i)$ of $\vec{X}_{m+1}(i)$ to the nearest neighbor point $\vec{X}_{m+1}^{N N}(i)$ is calculated:

$R_{m+1}(i)=\left\|\vec{X}_{m+1}(i)-\vec{X}_{m+1}^{N N}(i)\right\|$

(iii) The relationship between $E(m)$ and $m$ is shown:

$E(m)=\frac{1}{N-m \tau} \sum_{i=1}^{N-m \tau} \frac{\left\|\vec{X}_{m+1}(i)-\vec{X}_{m+1}^{N N}(i)\right\|}{\left\|\vec{X}_{m}(i)-\vec{X}_{m}^{N N}(i)\right\|}$

$E_{1}(m)=\frac{E(m+1)}{E(m)}$

(iv) Continue increasing the dimension of the phase space $m_{1}$, and make it larger than $m$, then repeat the calculation of the first three steps. Based on the results of each calculation, the relationship between $E_{1}\left(m_{i}\right)$ and $m_{i}$ is charted. While $E_{1}\left(m_{i}\right)$ in the graph reaches a plateau within a certain threshold, the corresponding $m_{i}$ is the optimal $m$ of the $\vec{X}_{m}(i)$.

(v) In practical applications, a criterion is defined to judge the smoothness of the finite sequence change of $E_{1}\left(m_{i}\right)$ and $m_{i}$, it is shown as:

$E^{*}(m)=\frac{1}{N-m \tau} \sum_{i=1}^{N-m \tau}\left\|\vec{X}_{m+1}(i)-\vec{X}_{m+1}^{N N}(i)\right\|$ 


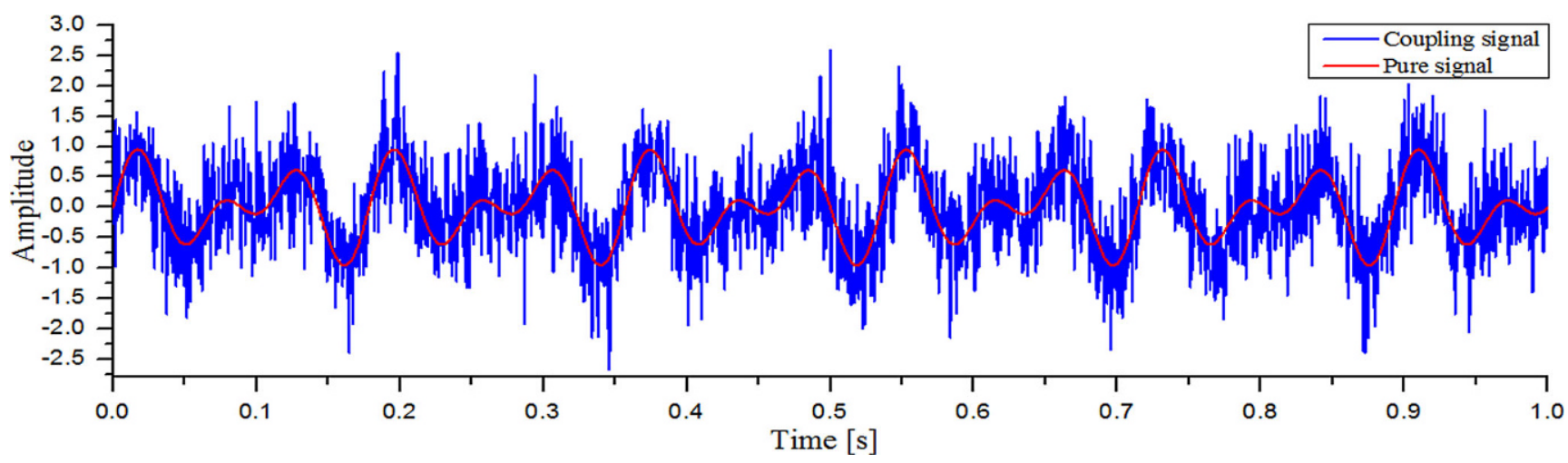

Fig. 3 Time domain diagram of coupled signals

$E_{2}(m)=\frac{E^{*}(m+1)}{E^{*}(m)}$

From the equations, in a range of $m_{i}$, if $E_{2}(m)=1$ the signal is random; if $E_{2}(m)$ within a certain threshold are not all equal to 1 , the signal is the deterministic signal.

\subsection{Evaluation of de-noising effect}

The definition of SNR is expressed, shown as in follow:

$S N R=10 \lg \left[\frac{\sum_{i=1}^{N} x^{2}(i)}{\sum_{i=1}^{N}[x(i)-z(i)]^{2}}\right]$

where, $z(i)$ is the de-noising signal.

The definition of RMSE for root-mean-square error evaluation is expressed, shown as in follow:

$R M S E=\sqrt{\frac{\sum_{i=1}^{N}[x(i)-z(i)]^{2}}{N}}$

where, $N$ is the length of $x(i)$.

Table 1 Input parameters of the simulation signal

\begin{tabular}{ll}
\hline Signal name & Parameters \\
\hline Pure signal $f_{\text {th }}(t)$ & $f_{\text {th }}(t)=\sin (28 \omega t) \cos (5.6 \omega t)$ \\
Random noise & The mean is 0 and the variance is 1 \\
$\xi_{G-n o i s e}(t)$ & \\
Pulse signal $\xi_{G \text {-pulse }}(t)$ & Frequency is $0.1 \mathrm{~Hz}$ \\
\hline
\end{tabular}

\section{Numerical simulation}

Fig. 2 is flowchart of the simulation for the signal de-noising. Firstly, the model of signal is based on the noise-free signal $f_{\text {th }}(t)$, the coupling signal $f_{0}(t)$ is formed by the combination of a random noise signal $\xi_{G \text {-noise }}(t)$ and impulse signal $\xi_{G \text {-pulse }}(t)$. Fig. 3 is the obtained time-domain diagram of the coupling signal after fitting simulations. And the expression of the simulation signal is shown as

$$
f_{0}(t)=f_{\text {th }}(t)+\xi_{G-\text { noise }}(t)+\zeta_{G \text {-pulse }}(t)
$$

The other parameters are shown in Table 1.

Base on the signal, the operation of de-nosing by the simulation is listed as:

(i) Determine $\tau$

Fig. 4 is the result of autocorrelation value by $\tau$. It is calculated based on $f_{0}(t)$ by the autocorrelation function. While $C(\tau)=1 / e$, the corresponding time value is the optimal $\tau$ of $f_{0}(t)$. From Fig. 4, the optimal $\tau=8$.

(ii) Determine $m$

Fig. 5 is the results of $m-E_{1}(m)$ and $m-E_{2}(m)$, which calculated by Eqs. 13 and 14. The blue line

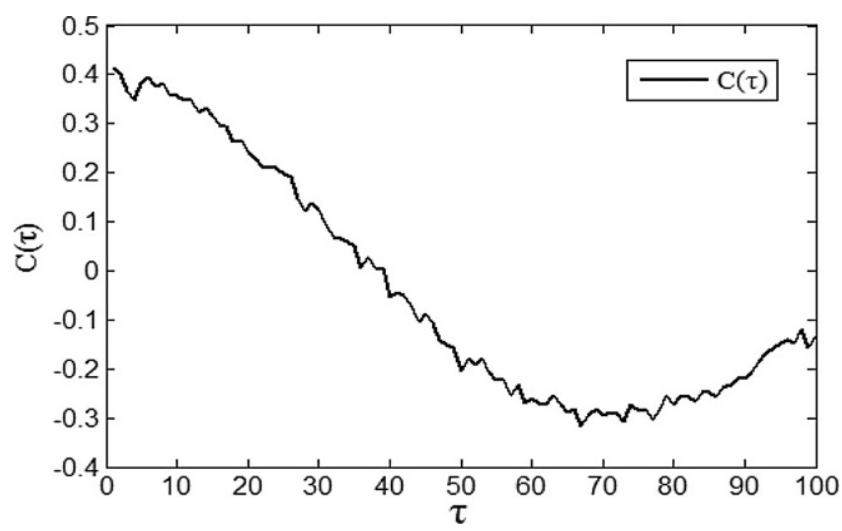

Fig. 4 The autocorrelation function 


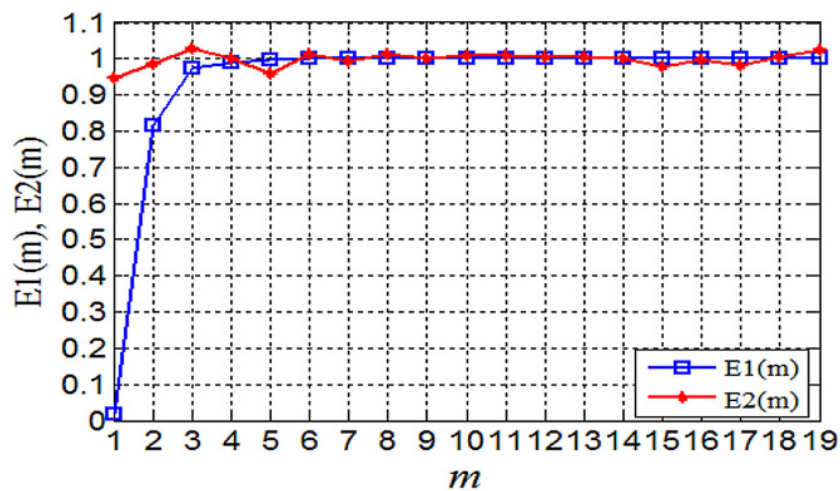

Fig. 5 The relationship between $E_{1}(m), E_{2}(m)$ and $m$ of simulation signals

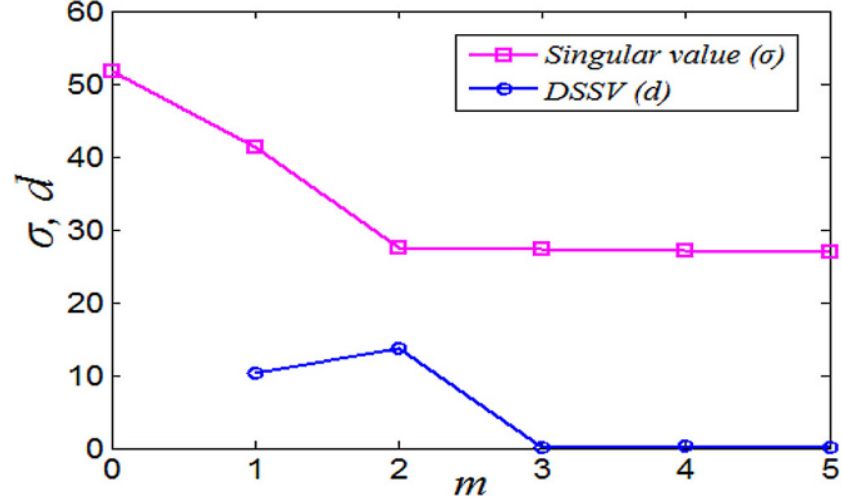

Fig. 6 Singular value and differential spectrum of signal

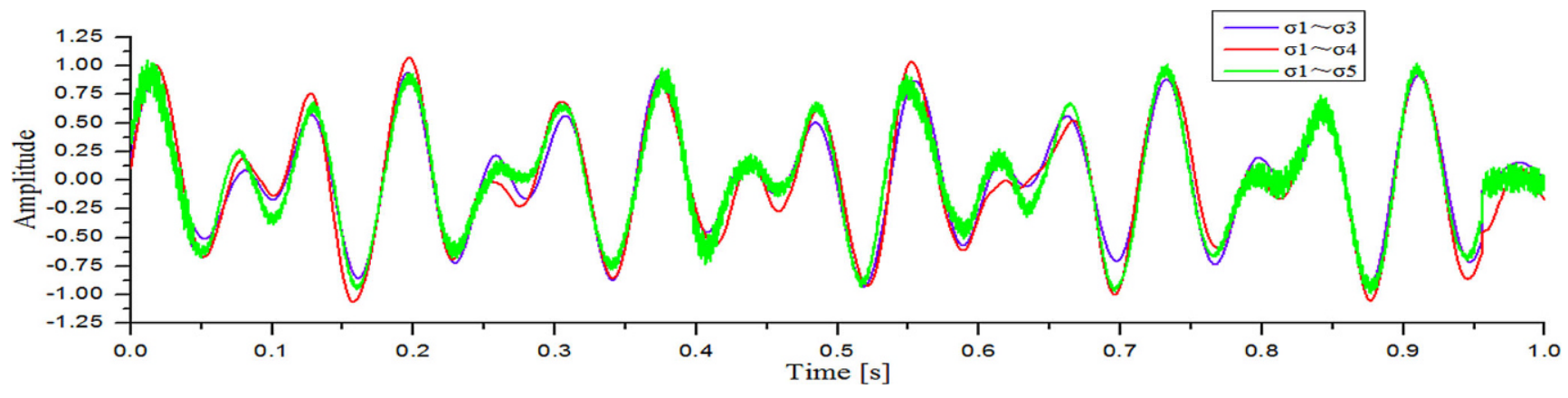

Fig. 7 Relationship between valid singular values and characteristic signals

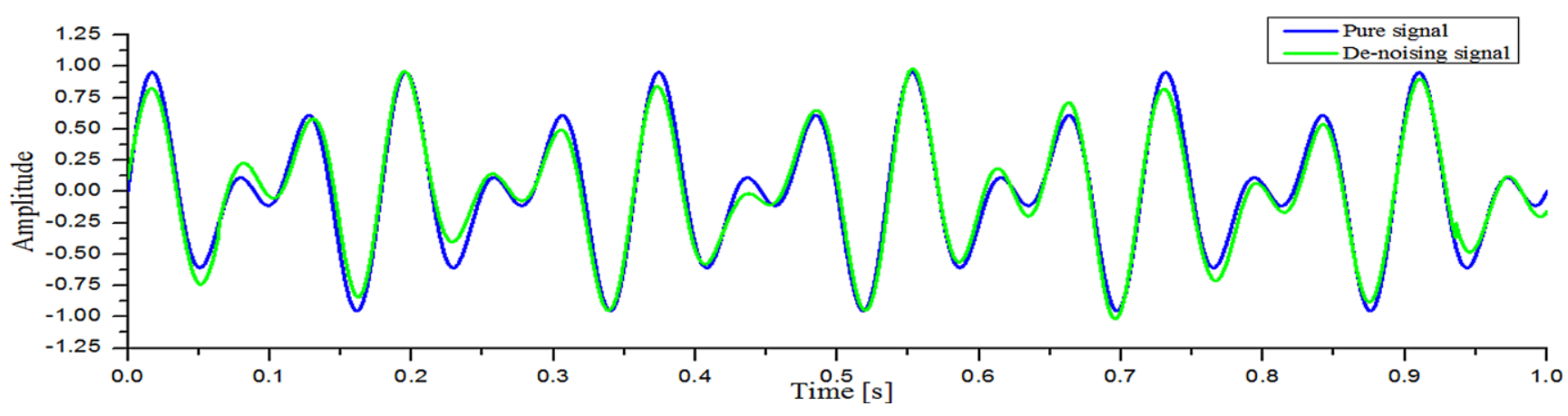

Fig. 8 Time domain diagram of the coupled signal after de-noising

shows that while $m$ varies from 1 to 4 , the value of $E_{1}(m)$ is rapidly approaching to 1 . While $m=5$, the value of $E_{1}(m)=1$ for the first time, and While $m>5$, $E_{1}(m)=1$. The red line shows that $E_{2}(m)=1$ in the range $1<m<19$ except the point of $m=1,2,3,5,15$, 17 , and 19 . The analysis above shows that the certain motion characteristics and the optimal value of the embedding dimension is $m=5$.

(iii) SVD of simulation signals

Fig. 6 is the difference spectrum and singular value by $m$, the first four singular values $\sigma_{1}=51.64, \sigma_{2}=41.26$, $\sigma_{3}=27.52$ and $\sigma_{4}=27.64$. The value of $\sigma_{3}$ is the singu- lar values mutates which separates the phase space of the effective signal and the noise signal. Meanwhile, in the singular value difference spectrum sequence, the first three large difference spectra are $d_{1}=10.39$, $d_{2}=13.74$, and $d_{3}=0.12$ respectively, the maximum difference spectrum is $d_{2}$.

(iv) Determine de-noising order Fig. 7 is the simulation results of the relationship between $\sigma$ and DSSV. In the case that $\sigma_{1}$ and $\sigma_{2}$ are selected for the reconstructed signal, many information is lost; in the case of selecting $\sigma_{1}, \sigma_{2}$ and $\sigma_{3}$, it contains a little noise information; in the case of 
Table 2 Evaluation of de-noising effect of simulation signal

\begin{tabular}{ll}
\hline SNR $(\mathrm{dB})$ & RMSE \\
\hline 31.31 & 0.34 \\
\hline
\end{tabular}

Table 3 The delay time at each measurement point

\begin{tabular}{lllll}
\hline Measurement point & I & II & III & IV \\
\hline Delay time $\tau$ & 8 & 8 & 8 & 8 \\
\hline
\end{tabular}

selecting $\sigma_{1}, \sigma_{2}, \sigma_{3}$, and $\sigma_{4}$, the reconstructed signal is distorted; In the case of selecting from $\sigma_{1}$ to $\sigma_{5}$, a large amount of noise information is mixed in the reconstructed signals. As a result, the selection of $\sigma_{1}$ $\sigma_{2}$ and $\sigma_{3}$ is suitable because the effective information of the obtained characteristic signal remains relatively completely and has a good effect of de-noising.

The signals are decomposed of $f_{0}(t)$ by the principle of the SVD method, and the $\sigma_{1}$ to $\sigma_{4}$ are obtained. Combined with Cao's algorithm, the optimum parameters are $m=5$ and $\tau=8$. Finally, as shown in Fig. 8, the inverse solution of singular value is used to reconstruct the effective characteristic signal after de-noising. From the figure the reconstructed effective signal is proximity to the pure signal, which indicates that ISVD has the potential for de-noising.

As shown in Table 2, after the de-noising by ISVD. And the $\mathrm{SNR}=31.31 \mathrm{~dB}, \mathrm{RMSE}=0.34$ are acquired by Eqs. 15 and 16. It reminds ISVD has a good de-noising potential.

\section{Experimental verification}

Fig. 9a is shown the experiment layout. Fig. 9b is the photograph of experiment. On the outer side of the gearbox, four measurement points are set as Points I, II, III and IV with four uniaxial acceleration sensors for the collection of vibration signal.

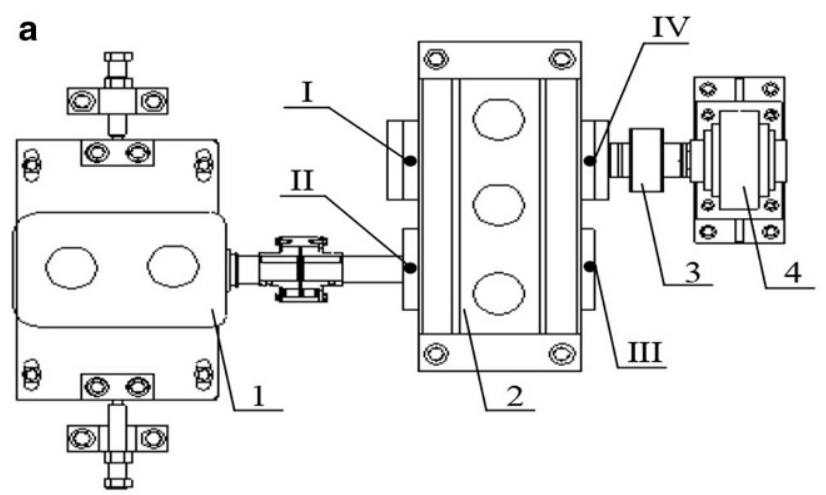

The speed of input is $1500 \mathrm{r} / \mathrm{min}$; the input current of the magnetic powder is $P=38 \mathrm{~W}$; the modulus of gear is $m_{o}=2 \mathrm{~mm}$. The gear material is $18 \mathrm{CrNiMo} 7$ steel.

\subsection{Experimental data}

Fig. 10 is shown the waveforms of vibration signal which were collected at four measurement points. The signals are affected by a large numbers of interference signals.

\subsection{Determination of $\tau$}

Table 3 is the calculation results. The minimum value of $\tau$ is $\tau=8$, which is calculated by the autocorrelation function method as while $\tau=8$ at each measurement point, the autocorrelation function $C(\tau) \approx 1 / e$.

\subsection{Determination of $m$}

Fig. 11 is the results of the computation of $m$ by the Cao's method in each measurement point. From Fig. 11a-d, in the range of $1 \leq m \leq 3$, while $m$ is increased, $E_{1}(m)$ is decreased as the domination of noise in the phase space. in the case of $m=3$, the minimum value of $E_{1}(m)$ is occurred as $E_{1}(m)=0.5$ in Points I, II and IV, and in Point III, the minimum is $E_{1}(m)=0.45$. The results indicate that noise content at measurement point III is the highest. While $3 \leq m \leq 6$, $E_{1}(m)$ is increases sharply with the increase of $m$. in the case of $m=6, E_{1}(m) \geq 0.8$, which indicates that the content of pseudo-nearest neighbor points of fault characteristics is decreased. While $m>6$, the value of $E_{1}(m)$ is slowly approached to 1 as $m$ is increased.

The value of $E_{2}(m)$ is used to determine the type of fault characteristic signals. In Fig. 11 , while $1 \leq m<6, E_{2}(m)$ is approached to 1 fluctuant, while $6 \leq m \leq 19$, the value of $E_{2}(m)$ is approximated to 1 , In addition, while $m=6$, the $E_{2}(m)$ value approaches constant value 1 for the first time. Indicating that there are certain vibration characteristics at

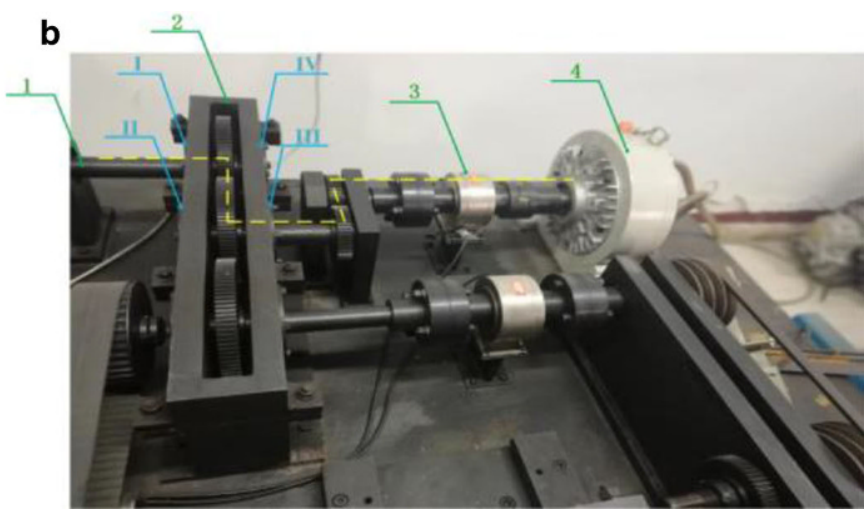

Fig. 9 Structural layout of the test bed. a Experimental schematic diagram. b Field diagram. 1. Input the motor and shaft; 2. Measured gear box; 3. Torque transducer; 4. Magnetic powder loader 
Fig. 10 Time domain diagram of vibration acceleration at points before de-noise
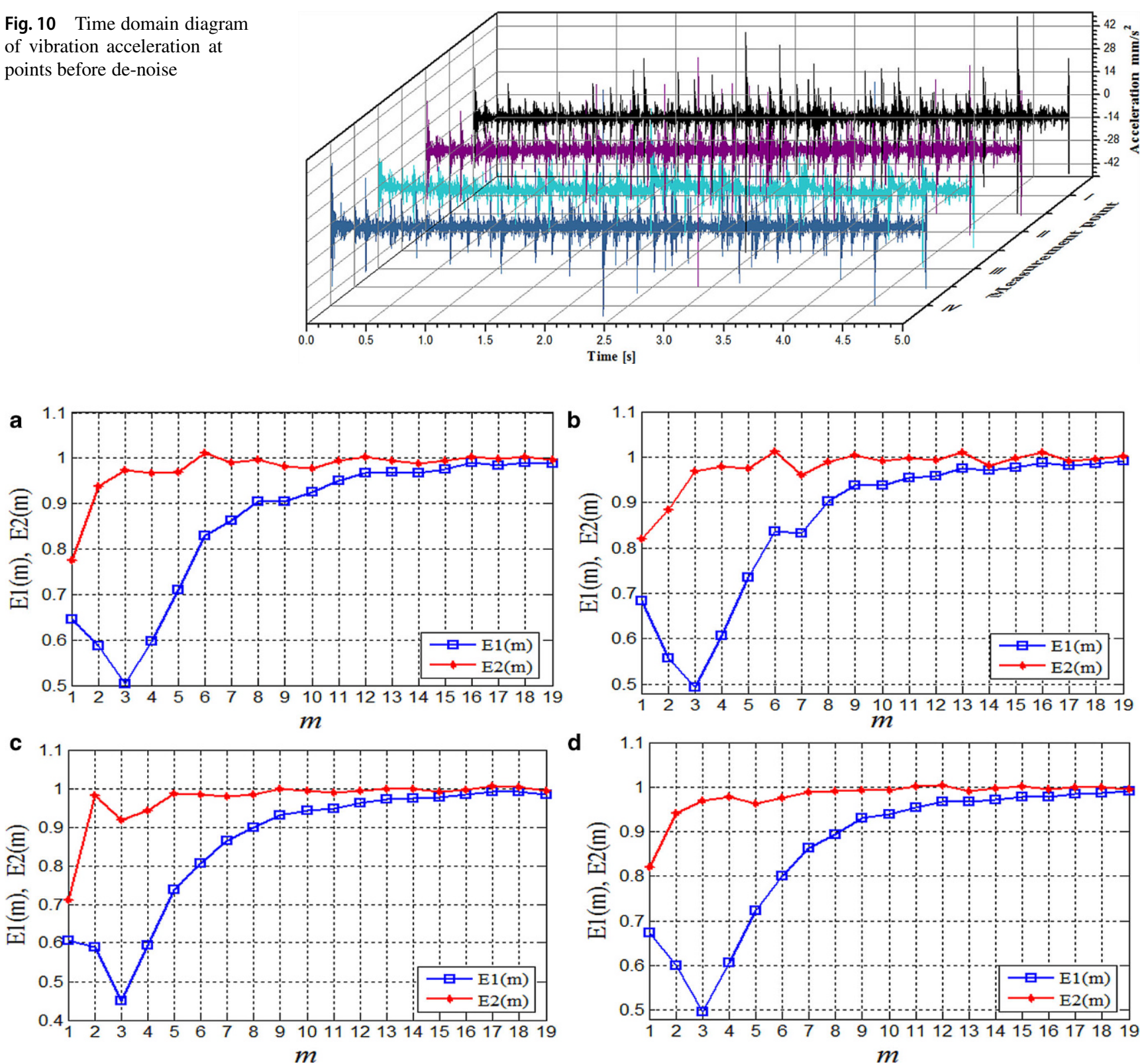

Fig. 11 Relationship between $E(m)$ and $m$ of experimental signals. a Measurement point I; b Measurement point II; c Measurement point III; d Measurement point IV

the four measuring points. It indicates that the fault characteristics are affected by random signals. At this point, $m$ is weak to the fault characteristics, which also makes the calculation of dimension meaningless. Finally, the optimal value of $m$ is $m=6$.

\subsection{The ISVD of experimental data}

Based on the calculation above, the parameters are set as: $\tau=8$ and $m=6$. Fig. 12 is the singular value of the experimental data which is decomposed at the measurement Points I, II, III, and IV by ISVD method. Fig. 12a is the singular value distribution graph. While $m<2$, the singular values of the four measurement points are $\sigma>800$, while $m>2$, the singular value in each point is smooth stability to a certain value as $\sigma_{\mathrm{I}}=900, \sigma_{\mathrm{II}}=900, \sigma_{\mathrm{III}}=700, \sigma_{\mathrm{IV}}=900$ the mutated point is $m=2$ or $m=3$. Fig. $12 \mathrm{~b}$ is the Singular value difference spectrum to supplement the determination of reconstruction order. In the figure, while $m=3,5$ and 6 , the difference spectrum values are approximately close to 50 . With the combination of results by Fig. 12a,b, the optimum reconstruction order is $\mathrm{DSSV}=3$. 
Table 4 Evaluation of de-noising effect of experimental data

\begin{tabular}{lll}
\hline Measurement point & SNR $(\mathrm{dB})$ & RMSE \\
\hline I & 46.29 & 0.31 \\
II & 46.47 & 0.12 \\
III & 45.41 & 0.33 \\
IV & 45.61 & 0.25 \\
\hline
\end{tabular}

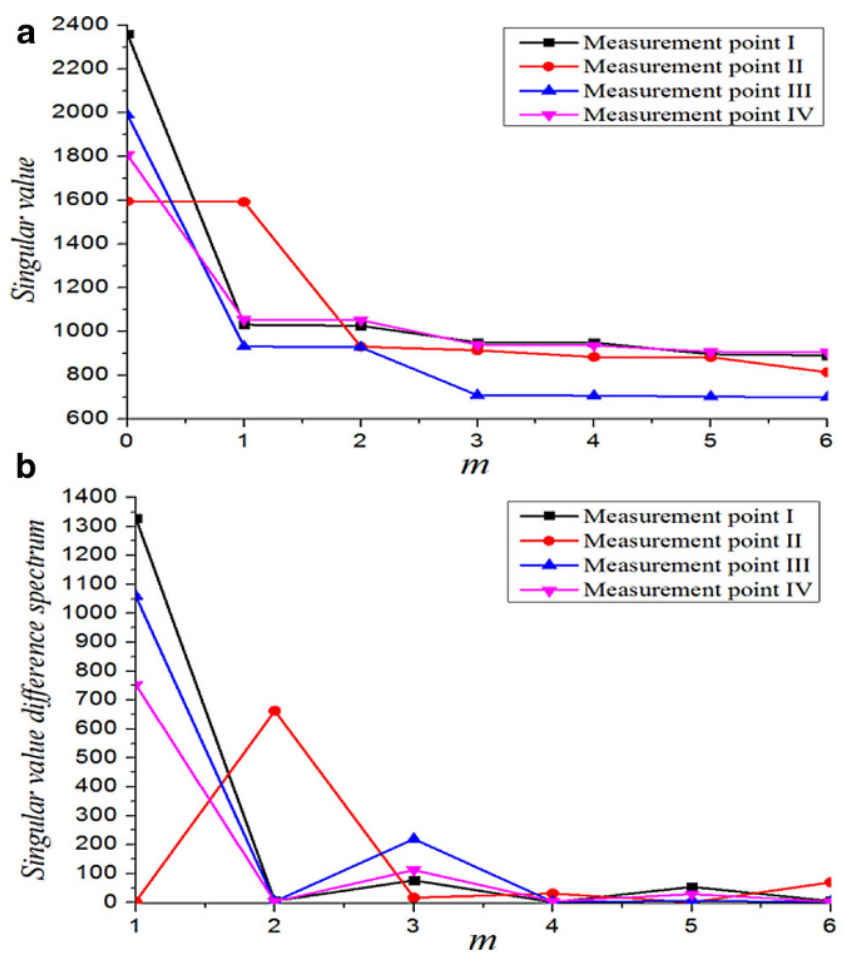

Fig. 12 The ISVD diagram of experimental data at each measurement point. a Singular value distribution graph; b Singular value difference spectrum

\subsection{Determine the de-noising order}

Fig. 13 is the reconstructed signal with fault characteristics of four measuring points in time-domain by Eq. 6. In the figures, the green wave is original signal, the brown wave is the de-noising signal. From the figures, the impact vibration generated by backlash and transmission error is dominant and effectively reduced after reconstruction. From the figures, the acceleration value of de-noising signal is much smaller than original one in each time. In addition, the trend of de-noising signal and original signal are same.

The effect of signal de-noising is evaluated by SNR method and RMSE, the results are showed in Table 4. From the results, SNR $\geq 45 \mathrm{~dB}$, and RMSE $<0.4$ in all measurement points. It reveals a better effect of de-noising by ISVD. In addition, it reveals that the de-noising effect of signals at Points I and II is better than Points III and IV.

\subsection{Experimental results and analysis}

Fig. 14 is the frequency response diagram of de-noising signals and original signals in each measurement point from Fig. 13 by Fourier transform. In Fig. 14, Fig. 14a,c and $\mathrm{d}$ are similar, because Point 1 Point III and Point IV are meshed. In the figures, the effective signals are retained in the frequency range of $300 \mathrm{~Hz}<f<800 \mathrm{~Hz}$ and $1.2 \mathrm{kHz}<f<1.7 \mathrm{kHz}$ as the different rotation speeds of each axis, signals of other frequencies are noise which is denoised by ISVD. Fig. 14b are the signals in Point II which near the input source and affected by more vibrations. In the figure, the signal of noise is de-noised in ineffective range of frequency, and the effective signal is retained.

\section{Conclusions}

In this research, a new method of ISVD is proposed for the vibration signal de-noising of gear pitting fault identification. Simulation and experiments have been conducted to prove the efficiency of ISVD. The conclusions are listed as follows:

(i) The theory of ISVD is expressed, the method for the determination of parameters $\tau$ and $m$ for SVD are optimized by autocorrelation function and Cao's algorithm respectively. The signal after de-noising is reconstructed by the inverse of the Hankel matrix.

(ii) In the simulation, the ISVD method is employed to de-noise the artificial vibration signal in a mathematical model of gear pitting fault, the results are $\mathrm{SNR}=31.3 \mathrm{~dB}$ and $\mathrm{RMSE}=0.34$. The simulation results reveal that ISVD has a good effect on de-noising of the fault vibration signal.

(iii) In the experiment, the ISVD method is adopted to de-noising the vibration signal of gear pitting fault identification, the results demonstrate SNR $>45 \mathrm{~dB}$, and RMSE $<0.4$. The experiment results reveal that ISVD has a good effect on de-noising of the fault vibration signal. 

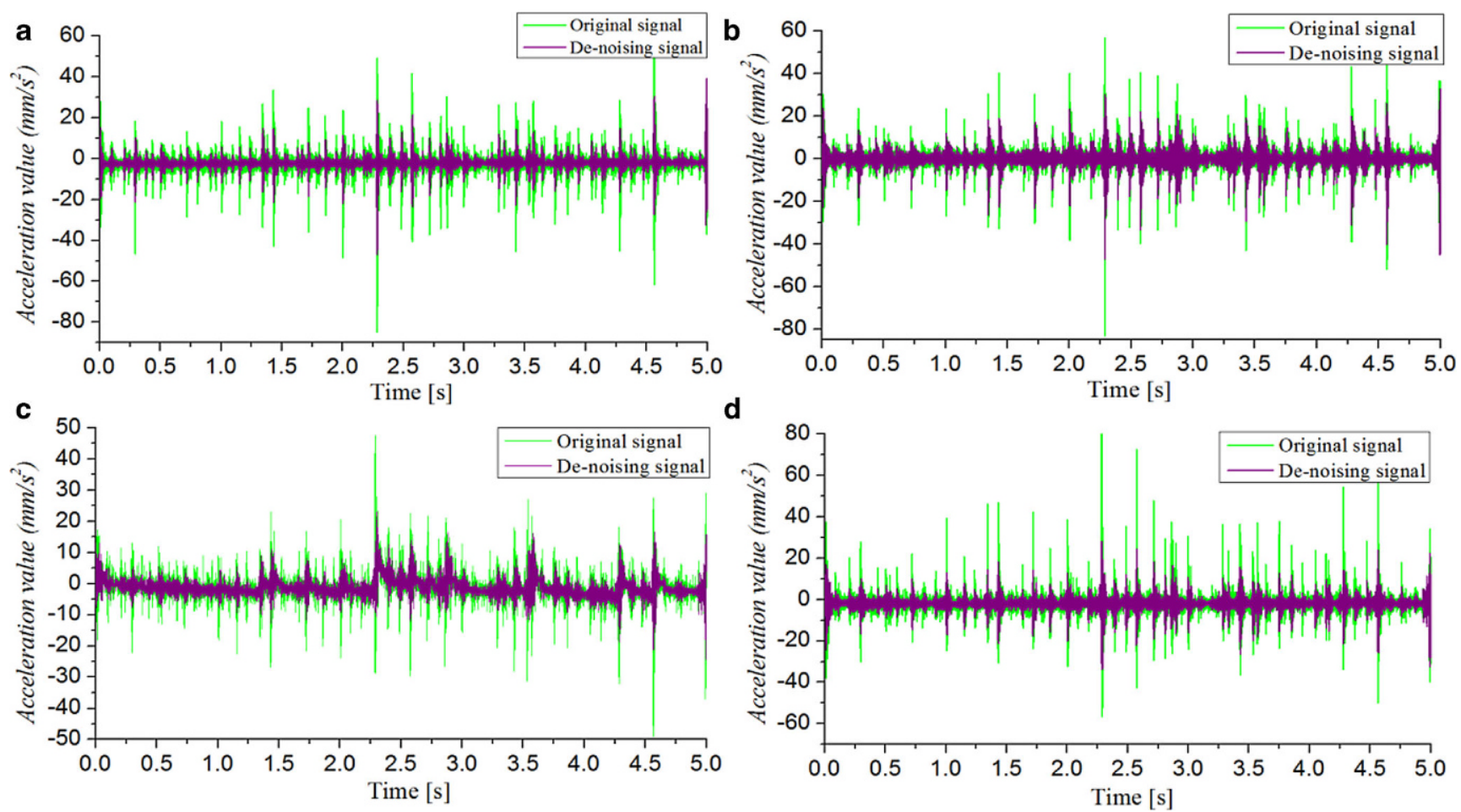

Fig. 13 Time domain diagram of vibration acceleration at measurement points after de-noising. a Measurement point I; b Measurement point II; c Measurement point III; d Measurement point IV
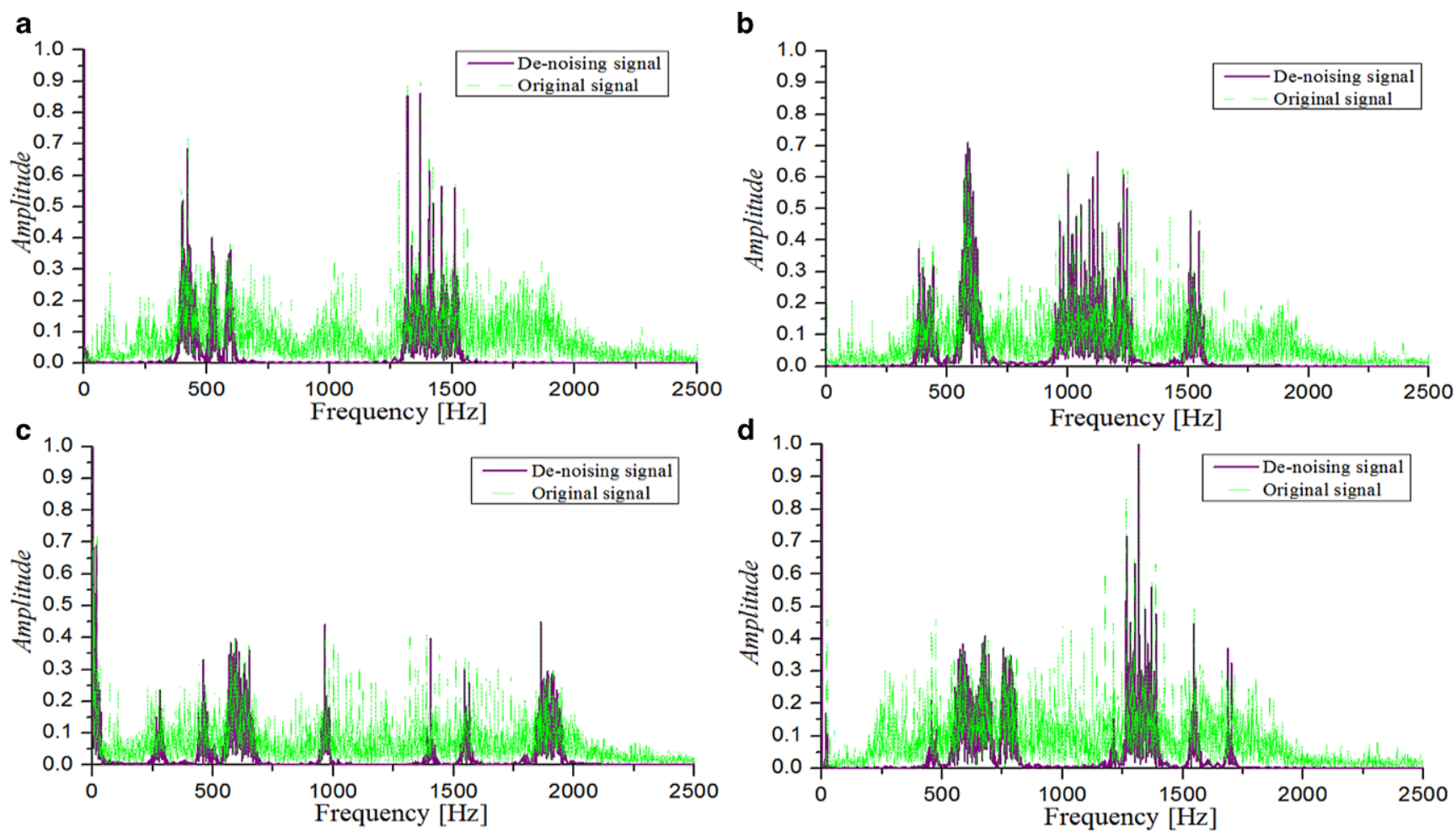

Fig. 14 Frequency response characteristics at each measurement point. a Measurement point I; $\mathbf{b}$ Measurement point II; $\mathbf{c}$ Measurement point III; d Measurement point IV 
Acknowledgements This work was supported partially by the National Natural Science Foundations of China (No.51175419) and Shaanxi Key Laboratory of Machinery Manufacturing Equipment Construction Project.

Open Access This article is licensed under a Creative Commons Attribution 4.0 International License, which permits use, sharing, adaptation, distribution and reproduction in any medium or format, as long as you give appropriate credit to the original author(s) and the source, provide a link to the Creative Commons licence, and indicate if changes were made. The images or other third party material in this article are included in the article's Creative Commons licence, unless indicated otherwise in a credit line to the material. If material is not included in the article's Creative Commons licence and your intended use is not permitted by statutory regulation or exceeds the permitted use, you will need to obtain permission directly from the copyright holder. To view a copy of this licence, visit http://creativecommons.org/licenses/by/4. $0 \%$.

\section{References}

1. Li Z, Li W, Zhao X (2019) Feature frequency extraction based on singular value decomposition and its application on rotor faults diagnosis. J Vib Control 25:1246-1262

2. Nandi S, Toliyat H, Li X (2005) Condition monitoring and fault diagnosis of electrical motors-a review. IEEE Trans Energy Convers 20:719-729

3. Bellini A, Filippetti F, Tassoni C et al (2008) Advances in diagnostic techniques for induction machines. Ieee Trans Ind Electron 55:4109-4126

4. Wang L, Cai G, Wang J et al (2018) Dual-enhanced sparse decomposition for wind turbine gearbox fault diagnosis. IEEE Trans Instrum Meas:1-12. https://doi.org/10.1109/TIM.2018.2851423

5. Huang N, Shen Z, Long S et al (1998) The empirical mode decomposition and the Hilbert spectrum for non-linear and nonstationary time series analysis. Proc R Soc Lond A Math Phys Sci 454:903-995

6. Tong S, Zhang Y, Xu J et al (2017) Pattern recognition of rolling bearing fault under multiple conditions based on ensemble empirical mode decomposition and singular value decomposition. J Mech Eng Sci 232:2280-2296

7. Smith J (2005) The local mean decomposition and its application to EEG perception data. J R Soc Interface 2:443-454

8. Philipp Z, Daniel F, Stephan R (2019) Active control of planetary gearbox vibration using phase-exact and narrowband simultaneous equations adaptation without explicitly identified secondary path models. Mech Syst Signal Process 120:234-251

9. Lei R, Shang P (2018) New irreversibility measure and complexity analysis based on singular value decomposition. Phys A Stat Mech Appl 212:913-924

10. Bie F, Horoshenkov K, Qian J et al (2019) An approach for the impact feature extraction method based on improved modal decomposition and singular value analysis. J Vib Control 5:1096-1108

11. Li C, Chen L, Tao L et al (2019) Features of singular value decomposition and its application to the vibration monitoring of turboprop engine. Commun Signal Process Syst 463:1495-1506

12. Shu X, Han S (2009) Improvement of DOA estimation using wavelet de-noising. The 1st International Conference on Information Science and Engineering, pp 587-590

13. Abdeldjalil O (2013) A review of wavelet de-noising in medical imaging. The 8th International Workshop on Systems, Signal Processing and their Applications (WoSSPA), pp 19-26

14. Golestani A, Kolbadi S, Heshmati A (2013) Localization and denoising seismic signals on SASW measurement by wavelet transform. J Appl Geophys 98:124-133
15. Cui Y, Li L, Chen R et al (2018) Incipient fault diagnosis of rolling bearing using accumulative component kurtosis in SVD process. J Vibroeng 20:1443-1458

16. Wei Y, Wei C, Feng R et al (2017) Study on rolling bearings fault feature extraction based on improved EEMD and SVD. J Shenyang Ligong Univ 36(106):99-102

17. Chen B, He Z, Chen X et al (2011) A demodulating approach based on local mean decomposition and its applications in mechanical fault diagnosis. Meas Sci Technol 22:55704-55716

18. Liu W, Zhang W, Han J et al (2012) A new wind turbine fault diagnosis method based on the local mean decomposition. Renew Energy 48:411-415

19. Yuan B, Chen Z, Xu S (2014) Micro-doppler analysis and separation based on complex local mean decomposition for aircraft with fast-rotating parts in ISAR imaging. IEEE Trans Geosci Remote Sens 52:1285-1298

20. He Q, Chen G, Chen X et al (2012) Application of singular value and wavelet de-noising method in analyzing vibration signal of piston pump. J Mech Strength 34:475-480

21. Duan L, Zhang L, Wang Z (2010) De-noising of reciprocating compressor vibration signal using wavelet packet and singular value based on gradient threshold. J Mech Strength 32:353-357

22. Li L, Cui Y, Chen R et al (2018) Optimal SES selection based on SVD and its application to incipient bearing fault diagnosis. Shock Vib :1-13. https://doi.org/10.1155/2018/8067416

23. Yang H, Lin H, Ding K (2018) Sliding window de-noising K-singular value decomposition and its application on rolling bearing impact fault diagnosis. J Sound Vib 421:205-219

24. Tang N, Tong S, Xu J et al (2018) Research on feature extraction based on short-time series reconstruction and K-SVD. Mach Des Res 34:18-22

25. Han M, Ren W, Xu M et al (2018) Nonuniform state space reconstruction for multivariate chaotic time series. IEEE Trans Cybern 49:1-11

26. Souza S, Rojas O (2018) Quasi-phases and pseudo-transitions in one-dimensional models with nearest neighbor interactions. Solid State Commun 269:131-134

27. Zhou X, Cui Y, Liu X, Li L, Wang L (2019) Fault identification technology for gear tooth surface wear based on MPE method by MI and improved FNN algorithm. Vibroeng Proced 28:24-29

28. Cao L, Wu S, Zhao H (1994) Chaos in topological Markov chains. Syst Sci Math Sci 7:97-105

29. Peña J, Sauer T (2019) SVD update methods for large matrices and applications. Linear Algebra Appl 561:41-62

30. Merainani B, Rahmoune C, Benazzouz D et al (2017) A novel gearbox fault feature extraction and classification using Hilbert empirical wavelet transform, singular value decomposition, and SOM neural network. J Vib Control 24:2512-2531

31. Li H, Liu T, Wu X et al (2019) Research on bearing fault feature extraction based on singular value decomposition and optimized frequency band entropy. Mech Syst Signal Process 118:477-502

32. Ma J, Wu J, Wang X (2018) A hybrid fault diagnosis method based on singular value difference spectrum de-noising and local mean decomposition for rolling bearing. J Low Freq Noise Vib Act Control 37:928-954

33. Gong Z, Liu C, Teo K et al (2019) Distributionally robust parameter identification of a time-delay dynamical system with tochastic measurements. Appl Math Model 69:685-695

34. Zhao T, Liu J, Dian S (2019) Finite-time control for interval type-2 fuzzy time-delay systems with norm-bounded uncertainties and limited communication capacity. Inf Sci 483:153-173

35. Lu Y (2006) Research on nonlinear feature extraction of noises radiated from underwater targets, pp 1-149 (Harbin Engineering University)

36. Luo Y (2013) Comparative study on traffic flow prediction based on ESN and Elman neural networks. J Hunan Univ Technol 27:67-72 